Supporting information for

\title{
An integrative toolbox for synthetic biology in Rhodococcus
}

\author{
James W. Round, Logan D. Robeck, and Lindsay D. Eltis* \\ Department of Microbiology and Immunology, Life Sciences Institute, The University of British Columbia, \\ Vancouver, BC, Canada, V6T 1 Z3.
}

Running title: Synthetic biology tools for Rhodococcus

${ }^{*}$ To whom correspondence should be addressed: Lindsay D. Eltis, Department of Microbiology and Immunology, 2350 Health Sciences Mall, Life Sciences Institute, The University of British Columbia, Vancouver, BC, V6T 1Z3, Canada. Tel.: +1-604-822-0042; Fax: +1-604-822-6041; E-mail: leltis@mail.ubc.ca

Table S1. Bacterial strains used in this study.

\begin{tabular}{lll}
\hline Strains & Use & Reference \\
\hline Escherichia coli strains & & $\mathrm{n} / \mathrm{a}$ \\
E. coli DH5 $\alpha$ & Propagation of DNA & 1 \\
E. coli DH5 $\alpha$ pir & Propagation of DNA & 1 \\
E. coli DH5 $\alpha$ pir-116 & Propagation of DNA & \\
\hline Rhodococcus strains & & 2 \\
\hline R. jostii RHA1 & Wild-type, promoter characterization & \\
\hline
\end{tabular}


Table S2. Plasmids used in this study

\begin{tabular}{|c|c|c|}
\hline Plasmid & Description / Use & Reference \\
\hline pSYN-mCherry & $\begin{array}{l}\text { High-copy modular integrative vector. Promotor-less backbone } \\
\text { carrying } m \text { Cherry. Cloning template. }\end{array}$ & 3 \\
\hline pSYN-P $(x)-m$ Cherry & $\begin{array}{l}\text { High-copy modular integrative vector carrying mCherry under } \\
\text { various constitutive promoters. Cloning templates. }\end{array}$ & 3 \\
\hline pSEVA111 & Copy-controlled cloning backbone. Amp ${ }^{\mathrm{R}}$. & 4 \\
\hline pSEVA211 & Copy-controlled cloning backbone. Kan ${ }^{R}$. & 4 \\
\hline pSEVA311 & Copy-controlled cloning backbone. ChlR. & 4 \\
\hline pRIME-P(x)-mCherry & $\begin{array}{l}\text { pRIME carrying } m \text { Cherry under the control of } \mathrm{P}_{(\mathrm{x})} \text {. } \\
\text { Promoter characterization }\end{array}$ & This study \\
\hline pRIME-P ${ }_{\mathrm{MG}-\mathrm{OP}(\mathrm{X})}-\mathrm{m}$ Cherry & $\begin{array}{l}\text { pRIME carrying } m \text { Cherry under the control of } \mathrm{P}_{\mathrm{MG}-\mathrm{OP}(\mathrm{X})} \\
\text { promoters. Optimized promoter characterization. }\end{array}$ & This study \\
\hline pRIME-P ${ }_{\mathrm{MG}-\mathrm{TO}(\mathrm{x})}-\mathrm{m}$ Cherry & $\begin{array}{l}\text { pRIME carrying } m \text { Cherry under the control of } \mathrm{P}_{\mathrm{MG}-\mathrm{TO}(\mathrm{x})} \\
\text { promoters. Tetracycline inducible promoter characterization. }\end{array}$ & This study \\
\hline pTetR-mCherry & $\begin{array}{l}\text { Modular copy-controlled integrative vector. Carrying } \\
\text { constitutively expressed tet } R(B) \text { and promotor-less mCherry. } \\
\text { Promoter characterization }\end{array}$ & This study \\
\hline pTetR- $\mathrm{P}_{\mathrm{MG}-\mathrm{TO}(\mathrm{x})}-$ mCherry & $\begin{array}{l}\text { PTetR carrying constitutively expressed tet } R(B) \text { and } m \text { Cherry } \\
\text { under the control of } \mathrm{P}_{\mathrm{MG} \text {-To }} \text { promoters. Tetracycline inducible } \\
\text { promoter characterization. }\end{array}$ & This study \\
\hline pRMvdh2 & $\begin{array}{l}\text { pRIME carrying } v d h \text { under the control of } \mathrm{P}_{\mathrm{T} 1} \text {. } \\
\text { Strain engineering. }\end{array}$ & This study \\
\hline $\mathrm{pLZ}$ & Zero-level uLoop recipient plasmid. & This study \\
\hline $\mathrm{pLO}(1: 4)$ & Odd-level uLoop recipient plasmids, 1 through 4 . & This study \\
\hline pLE1 & Even-level uLoop recipient plasmids, 1 . & This study \\
\hline pLEJR1 & $\begin{array}{l}\text { Even-level uLoop recipient plasmid } 1 \text { containing an integrative } \\
\text { multi-reporter cassette. Construct details in Table S7. }\end{array}$ & This study \\
\hline
\end{tabular}


Table S3. Primers used in this study

\begin{tabular}{|c|c|}
\hline Name & Sequence $\left(5^{\prime}\right.$ to $\left.3^{\prime}\right)$ \\
\hline \multicolumn{2}{|c|}{ Cloning Primers used to amplify indicated gene or fragment } \\
\hline$\varphi C 31 \_f w d$ & GTCCCCAATAATTACGATTTAAATGACGCCCCTCTATGGCCC \\
\hline$\varphi C 31$ rev & AGGATCTAGGGCGAGAGCTGCATCAGGTC \\
\hline ApraR_fwd & CAGCTCTCGCCCTAGATCCTTTTTGGTTCATG \\
\hline ApraR_rev & ATTAGGCGGGCTACTAATTTAAATGAGCTGAAGAAAGACAATCC \\
\hline mCherry-MCS_fwd & TTTTATTTGATGCCTTTAATCGCCGATGGTTTCTACAAAG \\
\hline mCherry-MCS_rev & CTATCAACAGGAGTCCAAGAGAAGAGCGCCCAATACGC \\
\hline Vdh2_fwd & CTTTAAGAAGGAGATATACATATGACTGACCTTCTCGG \\
\hline Vdh2_rev & CACGGGTGCCGGTGGGTCGAACTAGTCAGAACGGGAAC \\
\hline \multicolumn{2}{|c|}{ Promoter amplification primers } \\
\hline Pnit_fwd & GGTCGACTCTAGAGCGGCCGCTCACTCTTCTGCTCGGCC \\
\hline Pnit_rev & AACAAAATTATTTCTAGACGATATCGCCGTCCATTATACCTCCTCACGTGACGTGG \\
\hline PT1_fwd & GGTCGACTCTAGAGCGGCCGCACCGCTCTGGTCAGCGAC \\
\hline PT1_rev & AACAAAATTATTTCTAGACGATATCCTTGGGACGAAAGGAACTC \\
\hline PT2_fwd & GGTCGACTCTAGAGCGGCCGCACCGCTCTGGTCAGCGAC \\
\hline PT2_rev & AACAAAATTATTTCTAGACGATATCGAAAGGAACTCTACAACAGCGAC \\
\hline \multicolumn{2}{|l|}{$\underline{\text { uLoop primers }}$} \\
\hline CDS-mCherry_fwd & GGCTACGCTCTTCATCCAATGGTGAGCAAGGGCGAGGAG \\
\hline CDS-mCherry_rev & GGCTACGCTCTTCCTCGAAGCTCACTTGTACAGCTCGTCC \\
\hline Pro-PT1_fwd & GGCTACGCTCTTCGTCCGGAGCTCCTCCTGGCGAGCCGA \\
\hline Pro-PT1_rev & GGCTACGCTCTTCGTCGAGTACCTTGCGACGAAAGGAACTCTACAACAGC \\
\hline Pro-PT2_fwd & GGCTACGCTCTTCATCCGGAGCTCCTCCTGGCGAGCCGA \\
\hline Pro-PT2_rev & GGCTACGCTCTTCCTCGAGTACGAAAGGAACTCTACAACAGCGACACCTCAATG \\
\hline RBS-LG10_fwd a & $\begin{array}{l}\text { GGCTACGCTCTTCGTCCTACTGAAATAATTTTGTTTAACTTTAAAGAAGGAGATATAC } \\
\text { TAATGCGACGAAGAGCGTAGCC }\end{array}$ \\
\hline RBS-LG10_rev a & $\begin{array}{l}\text { GGCTACGCTCTTCGTCGCATTAGTATATCTCCTTCTTAAAGTTAAACAAAATTATTT } \\
\text { CAGTAGGACGAAGAGCGTAGCC }\end{array}$ \\
\hline Term-TthcA_fwd & GGCTACGCTCTTCATCCGCTTGAACTAGTCGACCCACCG \\
\hline Term-TthcA_rev & GGCTACGCTCTTCCTCGAGCGACTAGAGTCCCGCTGAGG \\
\hline
\end{tabular}

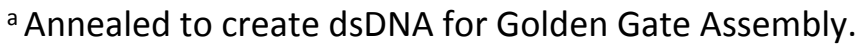


Table S4. Synthesized DNA and promoter features a,f

\section{Gene / Fragment}

tetR(B) expression cassette ${ }^{b}$

CAGGCTGCGCAACTGTTGGGAAGGGCGATCGACTAGAGTCCCGCTGAGGCGGCGTAGCAGGTCAGCCGCCCCAGCGGTGGTCACCAACCGGGGTGGAACGGC GCCGGTATCGGGTGTGTCCGTGGCGCTCATTCCAACCTCCGTGTGTTTGTGCAGGTTTCGCGTGTTGCAGTCCCTCGCACCGGCACCCGCAGCGAGGGGCTCACG GGTGCCGGTGGGTCGATCAGCTGCCGGACTCGCACTTCAGCTGCTTCTCGAGGCCGCAGATGATCAGTTCCAGGCCGAACAGGAAGGCCGGCTCGGCACCCTG GTGGTCGAACAGCTCGATGGCCTGCCGGAGCAGCGGCGGCATCGAGTCGGTGGTCGGCGTCTCGCGCTCCTCCTTCGCGACCTGGTGCTCCTGGTCCTCGAGGA CGCAGCCCAGGGTGAAGTGCCCGACGGCGGACAGGGCGTAGAGCGCGTTCTCCAGCGAGAAGCCCTGCTGGCACAGGAACGCGAGCTGGTTCTCGAGGGTCT CGTACTGCTTCTCGGTGGGACGGGTGCCGAGGTGCACCTTGGCGCCGTCGCGGTGCGACAGCAGCGCGCAGCGGAACGACTTGGCGTTGTTGCGGAGGAAGTC CTGCCACGACTCGCCCTCGAGCGGGCAGAAGTGGGTGTGGTGCCGGTCGAGCATCTCGATCGCAAGGGCGTCGAGTAACGCGCGCTTGTTCTTGACGTGCCAGT ACAGCGTCGGCTGCTCGACACCGAGCTTCTGCGCCAGCTTGCGGGTGGTGAGCCCTTCGATGCCGACCTCGTTCAGGAGTTCGAGTGCGGAGTTGATGACCTTG GACTTATCTAAGCGCGACATTGTATATCTCCTTCTTAAAGTTAAACAAAATTATTTCTAGACGGTACCGGCAGTTCATCCTCTCCCGCACTTCGTGAACTCTTTCCTT GCGACGAAAGGAACTCTACAACAGCGACACCTCAATGTCTACACTCGCCACTGCAGAAATTCTGGAGTCGGTTGACCAGGGATTTCATACGCGGCAGGGGCGAG TGGGTTCCGTCGACGTCGCCGGGTGGTCGCTCGACGGATCGGCTCGCCAGGAGGAGTTAATTAACGATCGGTGCGGGCCTCTTCGCTATTACGCC

\section{$P_{\text {tet }}{ }^{c}$}

CTGCAGGTCGACTCTAGAGCGGCCGCTTAAACTCTAGAAATATTGGATCGTCGGCACCGTCACGGCCGTGGGAGGCGGCACGATCCGCGACGTGATGATTCTGA CCAGGGAAAATAGCCCTCTGACCTGGGGATTTGACTTCCCTATCAGTGATAGAGATAATCTGGGAGTCCCTATCAGTGATAGAGAAGGCGGGATATCGTCTAGA AATAATTTTGT

$P_{\text {const }}{ }^{c}$

CTGCAGGTCGACTCTAGAGCGGCCGCTTAAACTCTAGAAATATTGGATCGTCGGCACCGTCACGGCCGTGGGAGGCGGCACGATCCGCGACGTGATGATCGGCC GCATCCCCACGGTGCTGCGCAGTGAGCTCTACGCCATCCCGGCGTTGATCTGTGCGTTCGCTGTGCGGGCTCTAACACGTCCTAGTATGGTAGGATGAGCAAGAT ATCGTCTAGAAATAATTTTGT

$P_{\text {amiM }}{ }^{c}$

CTGCAGGTCGACTCTAGAGCGGCCGCTTAAACTCTAGAAATATTGGATCGTCGGCACCGTCACGGCCGTGGGAGGCGGCACGATCCGCGACGTGATGATCGGCC GCATCCCCACGGTGCTGCGCAGTGAGCTCTACGCCATCCCGGCGTTGATCTGTGCGTTCGCAGAACGGGGCTTGTGGCCGTCCCTGTCGTGTGGTAAAATGTCCA CAAGATATCGTCTAGAAATAATTTTGT

$P_{n h M^{c}}$

CTGCAGGTCGACTCTAGAGCGGCCGCTTAAACTCTAGAAATATTGGATCGTCGGCACCGTCACGGCCGTGGGAGGCGGCACGATCCGCGACGTGATGATCGGCC GCATCCCCACGGTGCTGCGCAGTGAGCTCTACGCCATCCCGGCGTTGATCTGTGCGTTCGCTGTGCGGGCTCTAACACGTCCTAGTATGGTAGGATGAGCAAACA TCTACACATTGACATCCGTTCCGATGTGATGTAAAAATTGTCACGGATATCGTCTAGAAATAATTTTGT

$P_{\text {smyc }}{ }^{c}$

CTGCAGGTCGACTCTAGAGCGGCCGCTAATGATGATCGGCCGCATCCCCACGGTGCTGCGCAGTGAGCTCTACGCCATCCCGGCGTTGATCTGTGCGTTCGCACG CACAGGCCCGGTGTGAGAAGGGTCTCTGACGAGCGGGAGAACCCACCCGGGGTGGGCGAGTTTGTCCTGCGTGTGCTCGGTCGAGTAGGCTCTGGGAGATATC GTCTAGAAATAATTTTGT

$P_{\text {tac }}{ }^{c}$

CTGCAGGTCGACTCTAGAGCGGCCGCGTGCACCAATGCTTCTGGCGTCAGGCAGCCATCGGAAGCTGTGGTATGGCTGTGCAGGTCGTAAATCACTGCATAATT CGTGTCGCTCAAGGCGCACTCCCGTTCTGGATAATGTTTTTTGCGCCGACATCATAACGGTTCTGGCAAATATTCTGAAATGAGCTGTTGACAATTAATCATCGGC TCGTATAATGTGTGGAGATATCGTCTAGAAATAATTTTGT

$P_{\text {nit }}{ }^{g}$

GGTCGACTCTAGAGCGGCCGCTCACTCTTCTGCTCGGCCGAATCGAGCGCCGGCCGAATCGAGCGCCGGCCGAATCGAGCGCCGGCCGAATCGAGCGCCGGCC GAATCGAGCGCCGGCCGAATCGTTAGTGCGGTGTGCGTGCGTGGTGGTCGAGTATCGCGGCCCAGGAGGACGCTGGGAGCTTCTCGGGTGTACATATCGAGGC GGGCTCCCACGGCCGCCCGGGCTGAGGGAGCCGACGGCACGCGGCGGCTCACGGCGTGGCACGCGGAACGTCCGGGCTTGCACCTCACGTCACGTGAGGAGG TATAATGGACGGCGATATCGTCTAGAAATAATTTTGTT 
$P_{T 1} g$

GGTCGACTCTAGAGCGGCCGCACCGCTCTGGTCAGCGACCCGGACTGGCCCCACCGCGCCCGCGAGATCACGTCCAGCGCATCGGACTGGTAAGCCGGATTGG CTCAGTCACCTGGGGAGATGACACGTCCCCATCGTGGGCAGGGCTAAGGAGATGTTGTACCGATCCGCCTCCTCCTGGCGAGCCGATCCGTCGAGCGACCACCC GGCGACGTCGACGGAACCCACTCGCCCCTGCCGCGTATGAAATCCCTGGTCAACCGACTCCAGAATTTCTGCAGTGGCGAGTGTAGACATTGAGGTGTCGCTGTT GTAGAGTTCCTTTCGTCGCAAGGATATCGTCTAGAAATAATTTTGTT

\section{$P_{T 2}{ }^{g}$}

GGTCGACTCTAGAGCGGCCGCACCGCTCTGGTCAGCGACCCGGACTGGCCCCACCGCGCCCGCGAGATCACGTCCAGCGCATCGGACTGGTAAGCCGGATTGG CTCAGTCACCTGGGGAGATGACACGTCCCCATCGTGGGCAGGGCTAAGGAGATGTTGTACCGATCCGCCTCCTCCTGGCGAGCCGATCCGTCGAGCGACCACCC GGCGACGTCGACGGAACCCACTCGCCCCTGCCGCGTATGAAATCCCTGGTCAACCGACTCCAGAATTTCTGCAGTGGCGAGTGTAGACATTGAGGTGTCGCTGTT GTAGAGTTCCTTTCGATATCGTCTAGAAATAATTTTGTT

\section{$P_{M 6}{ }^{c}$}

CTGCAGGTCGACTCTAGAGCGGCCGCCTCCTCCTGGCGAGCCGATCCGTCGAGCGACCACCCGGCGACGTCGACGGAACCCACTCGCCCCTGCCGCGTATGAAA TCCCTGGTCAACCGACTCCAGAATTTCTGCAGTGGCGAGTGTAGACATTGAGGTGTCGCTGTTGTAGAGTTCCTTTCGTCGCAAGGAAAGAGTTCACGAAGTGCG GGAGAGGATGAACTGCCGATATCGTCTAGAAATAATTTTGT

\section{$P_{M 6-O P 1^{C}}$}

CTGCAGGTCGACTCTAGAGCGGCCGCCTCCTCCTGGCGAGCCGATCCGTCGAGCGACCACCCGGCGACGTCGACGGAACCCACTCGCCCCTGCCGCGTATGAAA TCCCTGGTCAACCGACTCCAGAATTTCTGCAGTGGCGAGTGTAGACATTGAGGTGTCGCTGTTGTAGAGTTGGITTCGTCGCAAGGAAAGAGTTCACGAAGTGC GGGAGAGGATGAACTGCCGATATCGTCTAGAAATAATTTTGT

\section{$P_{M 6-O P 2}{ }^{C}$}

CTGCAGGTCGACTCTAGAGCGGCCGCCTCCTCCTGGCGAGCCGATCCGTCGAGCGACCACCCGGCGACGTCGACGGAACCCACTCGCCCCTGCCGCGTATGAAA TCCCTGGTCAACCGACTCCAGAATTTCTGCAGTGGCGAGTGTAGACATTGAGGTGTCGCTGTGGTAGAGTTCCTTTCGTCGCAAGGAAAGAGTTCACGAAGTGC GGGAGAGGATGAACTGCCGATATCGTCTAGAAATAATTTTGT

$P_{\text {M6-OP3 }}{ }^{\mathrm{C}}$

CTGCAGGTCGACTCTAGAGCGGCCGCCTCCTCCTGGCGAGCCGATCCGTCGAGCGACCACCCGGCGACGTCGACGGAACCCACTCGCCCCTGCCGCGTATGAAA TCCCTGGTCAACCGACTCCAGAATTTCTGCAGTGGCGAGTGTAGACATTGAGGTGTCGCTGTGGTAGAGTTGGTTTCGTCGCAAGGAAAGAGTTCACGAAGTGC GGGAGAGGATGAACTGCCGATATCGTCTAGAAATAATTTTGT

$P_{M 6-O P 4}{ }^{c}$

CTGCAGGTCGACTCTAGAGCGGCCGCCTCCTCCTGGCGAGCCGATCCGTCGAGCGACCACCCGGCGACGTCGACGGAACCCACTCGCCCCTGCCGCGTATGAAA TCCCTGGTCAACCGACTCCAGAATTTCTGCAGTGGCGAGTTGAGACATTGAGGTGTCGCTGTGGTAGAGTTGGTTTCGTCGCAAGGAAAGAGTTCACGAAGTGC GGGAGAGGATGAACTGCCGATATCGTCTAGAAATAATTTTGT

$P_{\text {M6-TO1 }}{ }^{\mathrm{C}}$

CTGCAGGTCGACTCTAGAGCGGCCGCCTCCTCCTGGCGAGCCGATCCGTCGAGCGACCACCCGGCGACGTCGACGGAACCCACTCGCCCCTGCCGCGTATGAAA TCCCTGGTCAACCGACTCCAGAATTTCTGCAGTGGCGAGTGTAGACATTGAACTCTATCATTGATAGAGTTGGTTTCGTCGCAAGGAAAGAGTTCACGAAGTGC GGGAGAGGATGAACTGCCGATATCGTCTAGAAATAATTTTGT

\section{$P_{\text {M6-TO2 }}{ }^{\mathrm{C}}$}

CTGCAGGTCGACTCTAGAGCGGCCGCCTCCTCCTGGCGAGCCGATCCGTCGAGCGACCACCCGGCGACGTCGACGGAACCCACTCGCCCCTGCCGCGTATGAAA TCCCTGGTCAACCGACTCCAGAATTTCTGCAGTGGCGAGTGTAGACATTGATCCCTATCAGTGATAGAGATGGITTCGTCGCAAGGAAAGAGTTCACGAAGTGC GGGAGAGGATGAACTGCCGATATCGTCTAGAAATAATTTTGT

$P_{\text {M6-TO3 }}{ }^{\mathrm{C}}$

CTGCAGGTCGACTCTAGAGCGGCCGCCTCCTCCTGGCGAGCCGATCCGTCGAGCGACCACCCGGCGACGTCGACGGAACCCACTCGCCCCTGCCGCGTATGAAA TCCCTGGTCAACCGACTCCAGAATTTCTGCAGTGGCGAGTGTACTCTATCATTGATAGAGTTGGTAGAGTTGGTTTCGTCGCAAGGAAAGAGTTCACGAAGTGC GGGAGAGGATGAACTGCCGATATCGTCTAGAAATAATTTTGT 
$P_{\mathrm{MG}-\mathrm{TO} 4}{ }^{\mathrm{C}}$

CTGCAGGTCGACTCTAGAGCGGCCGCCTCCTCCTGGCGAGCCGATCCGTCGAGCGACCACCCGGCGACGTCGACGGAACCCACTCGCCCCTGCCGCGTATGAAA TCCCTGGTCAACCGACTCCAGAATTTCTGCAGTGGCGAGTGTTCCCTATCAGTGATAGAGATGGTAGAGTTGITTCGTCGCAAGGAAAGAGTTCACGAAGTGC GGGAGAGGATGAACTGCCGATATCGTCTAGAAATAATTTTGT

\section{$P_{\text {MG-TO5 }}{ }^{\mathrm{C}}$}

CTGCAGGTCGACTCTAGAGCGGCCGCCTCCTCCTGGCGAGCCGATCCGTCGAGCGACCACCCGGCGACGTCGACGGAACCCACTCGCCCCTGCCGCGTATGAAA TCCCTGGTCAACCGACACTCTATCATTGATAGAGTCGAGTGTAGACATTGAACTCTATCATTGATAGAGTTGGITTCGTCGCAAGGAAAGAGTTCACGAAGTGC GGGAGAGGATGAACTGCCGATATCGTCTAGAAATAATTTTGT

$P_{\text {M6-TO6 }}{ }^{\mathrm{C}}$

CTGCAGGTCGACTCTAGAGCGGCCGCCTCCTCCTGGCGAGCCGATCCGTCGAGCGACCACCCGGCGACGTCGACGGAACCCACTCGCCCCTGCCGCGTATGAAA TCCCTGGTCAACCGACTCCAGAATTTCTGCAGTGGCGAGTGTAGACATTGAACTCTATCATTGATAGAGTTGGTTTCGTCGCAAGGAAAGACTCTATCATTGATA GAGTAGGATGAACTGCCGATATCGTCTAGAAATAATTTTGT

$P_{\text {M6-TO7 }}{ }^{\mathrm{C}}$

CTGCAGGTCGACTCTAGAGCGGCCGCCTCCTCCTGGCGAGCCGATCCGTCGAGCGACCACCCGGCGACGTCGACGGAACCCACTCGCCCCTGCCGCGTATGAAA TCCCTGGTCAACCGACTCCCTATCAGTGATAGAGACGAGTGTAGACATTGAACTCTATCATTGATAGAGTTGGTTTCGTCGCAAGGAAAGAGTTCACGAAGTGC GGGAGAGGATGAACTGCCGATATCGTCTAGAAATAATTTTGT

$P_{M 6-T O 8}{ }^{c}$

CTGCAGGTCGACTCTAGAGCGGCCGCCTCCTCCTGGCGAGCCGATCCGTCGAGCGACCACCCGGCGACGTCGACGGAACCCACTCGCCCCTGCCGCGTATGAAA TCCCTGGTCAACCGACTCCAGAATTTCTGCAGTGGCGAGTGTAGACATTGAACTCTATCATTGATAGAGTTGGTTTCGTCGCAAGGAAAGTCCCTATCAGTGATA GAGAAGGATGAACTGCCGATATCGTCTAGAAATAATTTTGT

$P_{\text {MG-TO9 }}{ }^{\mathrm{C}}$

CTGCAGGTCGACTCTAGAGCGGCCGCCTCCTCCTGGCGAGCCGATCCGTCGAGCGACCACCCGGCGACGTCGACGGAACCCACTCGCCCCTGCCGCGTATGAAA TCCCTGGTCAACCGACACTCTATCATTGATAGAGTCGAGTGTTCCCTATCAGTGATAGAGATGGTAGAGTTGGTTTCGTCGCAAGGAAAGAGTTCACGAAGTGC GGGAGAGGATGAACTGCCGATATCGTCTAGAAATAATTTTGT

$P_{\text {M6-TO10 }}{ }^{c}$

CTGCAGGTCGACTCTAGAGCGGCCGCCTCCTCCTGGCGAGCCGATCCGTCGAGCGACCACCCGGCGACGTCGACGGAACCCACTCGCCCCTGCCGCGTATGAAA TCCCTGGTCAACCGACTCCAGAATTTCTGCAGTGGCGAGTGTTCCCTATCAGTGATAGAGATGGTAGAGTTGGITTCGTCGCAAGGAAAGACTCTATCATTGATA GAGTAGGATGAACTGCCGATATCGTCTAGAAATAATTTTGT

$P_{\text {M6-TO11 }}{ }^{\mathrm{C}}$

CTGCAGGTCGACTCTAGAGCGGCCGCCTCCTCCTGGCGAGCCGATCCGTCGAGCGACCACCCGGCGACGTCGACGGAACCCACTCGCCCCTGCCGCGTATGAAA TCCCTGGTCAACCGACTCCCTATCAGTGATAGAGACGAGTGTTCCCTATCAGTGATAGAGATGGTAGAGTTGGTTTCGTCGCAAGGAAAGAGTTCACGAAGTGC GGGAGAGGATGAACTGCCGATATCGTCTAGAAATAATTTTGT

$P_{\text {M6-TO12 }}{ }^{\mathrm{C}}$

CTGCAGGTCGACTCTAGAGCGGCCGCCTCCTCCTGGCGAGCCGATCCGTCGAGCGACCACCCGGCGACGTCGACGGAACCCACTCGCCCCTGCCGCGTATGAAA TCCCTGGTCAACCGACTCCAGAATTTCTGCAGTGGCGAGTGTTCCCTATCAGTGATAGAGATGGTAGAGTTGGITTCGTCGCAAGGAAAGTCCCTATCAGTGATA GAGAAGGATGAACTGCCGATATCGTCTAGAAATAATTTTGT 
Zero-level uLoop cassette ${ }^{d}$

TTTTATTTGATGCCTTTAATCATTACTCGCATCCATTCTCAGGCTGTCTCGTCTCGTCTCAGGTCTCCAGAAGAGCGCAGCTGGCACGACAGGTTTCCCGACTGGAA AGCGGGCAGTGAGCGCAACGCAATTAATGTGAGTTAGCTCACTCATTAGGCACCCCAGGCTTTACACTTTATGCTTCCGGCTCGTATGTTGTGTGGAATTGTGAG CGGATAACAATTTCACACAGGAAACAGCTATGACCATGATTACGCCAAGCTTGCATGCCTGCAGGTCGACTCTAGAGGATCCCCGGGTACCGAGCTCGAATTCAC TGGCCGTCGTTTTACAACGTCGTGACTGGGAAAACCCTGGCGTTACCCAACTTAATCGCCTTGCAGCACATCCCCCTTTCGCCAGCTGGCGTAATAGCGAAGAGG CCCGCACCGATCGCCCTTCCCAACAGTTGCGCAGCCTGAATGGCGAATGGCGCCTGATGCGGTATTTTCTCCTTACGCATCTGTGCGGTATTTCACACCGCATATG GTGCACTCTCAGTACAATCTGCTCTGATGCCGCATAGTTAAGCCAGCCCCGACACCCGCCAACACCCGCTGACGCGCCCTGACGGGCTTGTCTGCTCCCGGCATCC GCTTACAGACAAGCTGTGACGCTCTTCACGAGACCACCAGGATACATAGATTACCACAACTCCGAGCCCTTCCACCTCTTGGACTCCTGTTGATAG

\section{Odd-level 1 uLoop cassette ${ }^{d}$}

TTTTATTTGATGCCTTTAATCATTACTCGCATCCATTCTCAGGCTGTCTCGTCTCGTCTCCGAGCTCTTCAATGAGGAGTGAGACCATTGACAGCGCAGCTGGCACG ACAGGTTTCCCGACTGGAAAGCGGGCAGTGAGCGCAACGCAATTAATGTGAGTTAGCTCACTCATTAGGCACCCCAGGCTTTACACTTTATGCTTCCGGCTCGTA TGTTGTGTGGAATTGTGAGCGGATAACAATTTCACACAGGAAACAGCTATGACCATGATTACGCCAAGCTTGCATGCCTGCAGGTCGACTCTAGAGGATCCCCGG GTACCGAGCTCGAATTCACTGGCCGTCGTTTTACAACGTCGTGACTGGGAAAACCCTGGCGTTACCCAACTTAATCGCCTTGCAGCACATCCCCCTTTCGCCAGCT GGCGTAATAGCGAAGAGGCCCGCACCGATCGCCCTTCCCAACAGTTGCGCAGCCTGAATGGCGAATGGCGCCTGATGCGGTATTTTCTCCTTACGCATCTGTGCG GTATTTCACACCGCATATGGTGCACTCTCAGTACAATCTGCTCTGATGCCGCATAGTTAAGCCAGCCCCGACACCCGCCAACACCCGCTGACGCGCCCTGACGGG CTTGTCTGCTCCCGGCATCCGCTTACAGACAAGCTGTGACACTAGTGAGCACGGTCTCACGCTAGCATGAAGAGCACCCCAGGATACATAGATTACCACAACTCC GAGCCCTTCCACCTCTTGGACTCCTGTTGATAG

\section{Odd-level 2 uLoop cassette ${ }^{d}$}

TTTTATTTGATGCCTTTAATCATTACTCGCATCCATTCTCAGGCTGTCTCGTCTCGTCTCCGAGCTCTTCAGCAAGGAGTGAGACCATTGACAGCGCAGCTGGCACG ACAGGTTTTCCCGACTGGAAAGCGGGCAGTGAGCGCAACGCAATTAATGTGAGTTAGCTCACTCATTAGGCACCCCAGGCTTTACACTTTATGCTTCCGGCTCGTA TGTTGTGTGGAATTGTGAGCGGATAACAATTTCACACAGGAAACAGCTATGACCATGATTACGCCAAGCTTGCATGCCTGCAGGTCGACTCTAGAGGATCCCCGG GTACCGAGCTCGAATTCACTGGCCGTCGTTTTACAACGTCGTGACTGGGAAAACCCTGGCGTTACCCAACTTAATCGCCTTGCAGCACATCCCCCTTTCGCCAGCT GGCGTAATAGCGAAGAGGCCCGCACCGATCGCCCTTCCCAACAGTTGCGCAGCCTGAATGGCGAATGGCGCCTGATGCGGTATTTTCTCCTTACGCATCTGTGCG GTATTTCACACCGCATATGGTGCACTCTCAGTACAATCTGCTCTGATGCCGCATAGTTAAGCCAGCCCCGACACCCGCCAACACCCGCTGACGCGCCCTGACGGG CTTGTCTGCTCCCGGCATCCGCTTACAGACAAGCTGTGACACTAGTGAGCACGGTCTCACGCTATACTGAAGAGCACCCCAGGATACATAGATTACCACAACTCC GAGCCCTTCCACCTCTTGGACTCCTGTTGATAG

\section{Odd-level 3 uLoop cassette ${ }^{d}$}

TTTTATTTGATGCCTTTAATCATTACTCGCATCCATTCTCAGGCTGTCTCGTCTCGTCTCCGAGCTCTTCATACAGGAGTGAGACCATTGACAGCGCAGCTGGCACG ACAGGTTTCCCGACTGGAAAGCGGGCAGTGAGCGCAACGCAATTAATGTGAGTTAGCTCACTCATTAGGCACCCCAGGCTTTACACTTTATGCTTCCGGCTCGTA TGTTGTGTGGAATTGTGAGCGGATAACAATTTCACACAGGAAACAGCTATGACCATGATTACGCCAAGCTTGCATGCCTGCAGGTCGACTCTAGAGGATCCCCGG GTACCGAGCTCGAATTCACTGGCCGTCGTTTTACAACGTCGTGACTGGGAAAACCCTGGCGTTACCCAACTTAATCGCCTTGCAGCACATCCCCCTTTCGCCAGCT GGCGTAATAGCGAAGAGGCCCGCACCGATCGCCCTTCCCAACAGTTGCGCAGCCTGAATGGCGAATGGCGCCTGATGCGGTATTTTCTCCTTACGCATCTGTGCG GTATTTCACACCGCATATGGTGCACTCTCAGTACAATCTGCTCTGATGCCGCATAGTTAAGCCAGCCCCGACACCCGCCAACACCCGCTGACGCGCCCTGACGGG CTTGTCTGCTCCCGGCATCCGCTTACAGACAAGCTGTGACACTAGTGAGCACGGTCTCACGCTACAGTGAAGAGCACCCCAGGATACATAGATTACCACAACTCC GAGCCCTTCCACCTCTTGGACTCCTGTTGATAG

\section{Odd-level 4 uLoop cassette ${ }^{d}$}

TTTTATTTGATGCCTTTAATCATTACTCGCATCCATTCTCAGGCTGTCTCGTCTCGTCTCCGAGCTCTTCACAGAGGAGTGAGACCATTGACAGCGCAGCTGGCACG ACAGGTTTCCCGACTGGAAAGCGGGCAGTGAGCGCAACGCAATTAATGTGAGTTAGCTCACTCATTAGGCACCCCAGGCTTTACACTTTATGCTTCCGGCTCGTA TGTTGTGTGGAATTGTGAGCGGATAACAATTTCACACAGGAAACAGCTATGACCATGATTACGCCAAGCTTGCATGCCTGCAGGTCGACTCTAGAGGATCCCCGG GTACCGAGCTCGAATTCACTGGCCGTCGTTTTACAACGTCGTGACTGGGAAAACCCTGGCGTTACCCAACTTAATCGCCTTGCAGCACATCCCCCTTTCGCCAGCT GGCGTAATAGCGAAGAGGCCCGCACCGATCGCCCTTCCCAACAGTTGCGCAGCCTGAATGGCGAATGGCGCCTGATGCGGTATTTTCTCCTTACGCATCTGTGCG GTATTTCACACCGCATATGGTGCACTCTCAGTACAATCTGCTCTGATGCCGCATAGTTAAGCCAGCCCCGACACCCGCCAACACCCGCTGACGCGCCCTGACGGG CTTGTCTGCTCCCGGCATCCGCTTACAGACAAGCTGTGACACTAGTGAGCACGGTCTCACGCTAGGTTGAAGAGCACCCCAGGATACATAGATTACCACAACTCC GAGCCCTTCCACCTCTTGGACTCCTGTTGATAG

\section{Even-level 1 uLoop cassette ${ }^{d}$}

TTTTATTTGATGCCTTTAATCATTACTCGCATCCATTCTCAGGCTGTCTCGTCTCGTCTCCGAGGTCTCAGGAGAATGTGAAGAGCTTGACAGCGCAGCTGGCACG ACAGGTTTCCCGACTGGAAAGCGGGCAGTGAGCGCAACGCAATTAATGTGAGTTAGCTCACTCATTAGGCACCCCAGGCTTTACACTTTATGCTTCCGGCTCGTA TGTTGTGTGGAATTGTGAGCGGATAACAATTTCACACAGGAAACAGCTATGACCATGATTACGCCAAGCTTGCATGCCTGCAGGTCGACTCTAGAGGATCCCCGG GTACCGAGCTCGAATTCACTGGCCGTCGTTTTACAACGTCGTGACTGGGAAAACCCTGGCGTTACCCAACTTAATCGCCTTGCAGCACATCCCCCTTTCGCCAGCT GGCGTAATAGCGAAGAGGCCCGCACCGATCGCCCTTCCCAACAGTTGCGCAGCCTGAATGGCGAATGGCGCCTGATGCGGTATTTTCTCCTTACGCATCTGTGCG GTATTTCACACCGCATATGGTGCACTCTCAGTACAATCTGCTCTGATGCCGCATAGTTAAGCCAGCCCCGACACCCGCCAACACCCGCTGACGCGCCCTGACGGG CTTGTCTGCTCCCGGCATCCGCTTACAGACAAGCTGTGACACTAGTGAGCACGCTCTTCAGGTATACTTGAGACCACCCCAGGATACATAGATTACCACAACTCCG AGCCCTTCCACCGTCTTGGACTCCTGTTGATAG 


\begin{abstract}
Codon-optimized SfGFP e
GGCTACGCTCTTCGTCCAATGAAACATTTAACAGGCAGCAAGGGGGAGGAACTATTCACGGGTGTCGTCCCAATCCTCGTCGAACTCGACGGCGACGTCAACGG GCACAAGTTCAGCGTCCGCGGTGAAGGTGAGGGAGACGCCACCAACGGCAAGCTCACCCTGAAATTCATCTGCACCACCGGGAAACTGCCCGTCCCGTGGCCG ACCCTCGTAACTACTCTCACCTACGGCGTCCAGTGCTTCTCCCGCTACCCGGACCACATGAAGCAGCACGACTTCTTCAAGAGCGCGATGCCGGAGGGCTACGTC CAGGAGCGGACCATCAGCTTCAAGGACGACGGCACATACAAGACGAGGGCCGAGGTGAAGTTCGAGGGCGACACTCTGGTGAACCGGATCGAGCTGAAGGGC ATCGACTTCAAGGAGGACGGTAATATTCTCGGCCACAAGCTCGAGTACAACTTCAACTCGCACAACGTCTACATCACGGCCGACAAGCAGAAGAACGGCATCAA GGCCAACTTCAAGATCAGACACAACGTCGAGGACGGATCCGTTCAGCTGGCCGACCACTACCAGCAGAACACCCCGATCGGCGACGGGCCGGTGCTGTTGCCCG ACAACCACTACTTAAGCACCCAGTCGGTTCTGTCCAAGGACCCCAACGAGAAGCGGGATCACATGGTCCTGCTGGAATTCGTGACCGCGGCGGGCATAACGCAC GGCATGGACGAACTGTACAAGTGAGCTTCGATGAAGAGCGTAGCC
\end{abstract}

\title{
Domesticated $\phi C 31$ integrase fragment $1{ }^{e}$
}

GACGCCCCTCTATGGCCCGTACTGACGGACACACCGAAGCCCCGGCGGCAACCCTCAGCGGATGCCCCGGGGCTTCACGTTTTCCCAGGTCAGAAGCGGTTTTC GGGAGTAGTGCCCCAACTGGGGTAACCTTTGAGTTCTCTCAGTTGGGGGCGTAGGGTCGCCGACATGACACAAGGGGTTGTGACCGGGGTGGACACGTACGCG GGTGCTTACGACCGTCAGTCGCGCGAGCGCGAGAATTCGAGCGCAGCAAGCCCAGCGACACAGCGTAGCGCCAACGAAGACAAGGCGGCCGACCTTCAGCGC GAAGTCGAGCGCGACGGGGGCCGGTTCAGGTTCGTCGGGCATTTCAGCGAAGCGCCGGGCACGTCGGCGTTCGGGACGGCGGAGCGCCCGGAGTTCGAACGC ATCCTGAACGAATGCCGCGCCGGGCGGCTCAACATGATCATTGTCTATGACGTGTCGCGCTTCTCGCGCCTGAAGGTCATGGACGCGATTCCGATTGTCTCGGAA TTGCTCGCCCTGGGCGTGACGATTGTTTCCACTCAGGAAGGCGTCTTCCGGCAGGGAAACGTCATGGACCTGATTCACCTGATTATGCGGCTCGACGCGTCGCAC AAAGAATCTTCGCTGAAGTCGGCGAAGATTCTCGACACGAAGAACCTTCAGCGCGAATTGG

\section{Domesticated $\phi C 31$ integrase fragment $2 e$}

TTGGGCGGGTACGTCGGCGGGAAGGCGCCTTACGGCTTCGAGCTTGTTTCGGAGACGAAGGAGATCACGCGCAACGGCCGAATGGTCAATGTCGTCATCAACA AGCTTGCGCACTCGACCACTCCCCTTACCGGACCCTTCGAGTTCGAGCCCGACGTAATCCGGTGGTGGTGGCGTGAGATCAAGACGCACAAACACCTTCCCTTCA AGCCGGGCAGTCAAGCCGCCATTCACCCGGGCAGCATCACGGGGCTTTGTAAGCGCATGGACGCTGACGCCGTGCCGACCCGGGGCGAGACGATTGGGAAGA AGACCGCTTCAAGCGCCTGGGACCCGGCAACCGTTATGCGAATCCTTCGGGACCCGCGTATTGCGGGCTTCGCCGCTGAGGTGATCTACAAGAAGAAGCCGGAC GGCACGCCGACCACGAAGATTGAGGGTTACCGCATTCAGCGCGACCCGATCACGCTCCGGCCGGTCGAGCTTGATTGCGGACCGATCATCGAGCCCGCTGAGTG GTATGAGCTTCAGGCGTGGTTGGACGGCAGGGGGCGCGGCAAGGGGCTTTCCCGGGGGCAAGCCATTCTGTCCGCCATGGACAAGCTGTACTGCGAGTGTGGC GCCGTCATGACTTCGAAGCGCGGGGAAGAATCGATCAAGGACTCTTACCGCTGCCGTCGCCGGAAGGTGGTCGACCCGTCCG

\section{Domesticated $\phi C 31$ integrase fragment $3 e$}

TCCGCACCTGGGCAGCACGAAGGCACGTGCAACGTCAGCATGGCGGCACTCGACAAGTTCGTTGCGGAACGCATCTTCAACAAGATCAGGCACGCCGAAGGCG ACGAAGAGACGTTGGCGCTTCTGTGGGAAGCCGCCCGACGCTTCGGCAAGCTCACTGAGGCGCCTGAAAAGAGCGGCGAACGGGCGAACCTTGTTGCGGAGC GCGCCGACGCCCTGAACGCCCTTGAGGAGCTGTACGAAGACCGCGCGGCAGGCGCGTACGACGGACCCGTTGGCAGGAAGCACTTCCGGAAGCAACAGGCAG CGCTGACGCTCCGGCAGCAAGGGGCGGAGGAGCGGCTTGCCGAACTTGAAGCCGCCGAAGCCCCGAAGCTTCCCCTTGACCAATGGTTCCCCGAAGACGCCGA CGCTGACCCGACCGGCCCTAAGTCGTGGTGGGGGCGCGCGTCAGTAGACGACAAGCGCGTGTTCGTCGGCCTCTTCGTAGACAAGATCGTTGTCACGAAGTCGA CTACGGGCAGGGGGCAGGGAACGCCCATCGAGAAGCGCGCTTCGATCACGTGGGCGAAGCCGCCGACCGACGACGACGAAGACGACGCCCAGGACGGCACG GAAGACGTAGCGGCGTAGCGAGACACCCGGGAAGCCTGATCTACGTCTGTCGAGAAGTTTCTGATCGAAAAGTTCGACAGCGTCTCCGACCTGATGCAGCTCTC GC

\section{Domesticated aac(3)-IV, apramycin resistance marker $e^{2}$}

CTCCGAGGTCTCAGGAGCCTAGATCCTTTTGGTTCATGTGCAGCTCCATCAGCAAAAGGGGATGATAAGTTTATCACCACCGACTATTTGCAACAGTGCCGTTGAT CGTGCTATGATCGACTGATGTCATCAGCGGTGGAGTGCAATGTCGTGCAATACGAATGGCGAAAAGCCGAGCTCATCGGTCAGCTTCTCAACCTTGGGGTTACCC CCGGCGGTGTGCTGCTGGTCCACAGCTCCTTCCGTAGCGTCCGGCCCCTCGAAGATGGGCCACTTGGACTGATCGAGGCCCTGCGTGCTGCGCTGGGTCCGGGA GGGACGCTCGTCATGCCCTCGTGGTCAGGTCTGGACGACGAGCCGTTCGATCCTGCCACGTCGCCCGTTACACCGGACCTTGGAGTTGTCTCTGACACATTCTGG CGCCTGCCAAATGTAAAGCGCAGCGCCCATCCATTTGCCTTTTGCGGCAGCGGGGCCACAGGCAGAGCAGATCATCTCTGATCCATTGCCCCTGCCACCTCACTCG CCTGCAAGCCCGGTCGCCCGTGTCCATGAACTCGATGGGCAGGTACTTCTCCTCGGCGTGGGACACGATGCCAACACGACGCTGCATCTTGCCGAGTTGATGGC AAAGGTTCCCTATGGGGTGCCGAGACACTGCACCATTCTTCAGGATGGCAAGTTGGTACGCGTCGATTATCTCGAGAATGACCACTGCTGTGAGCGCTTTGCCTT GGCGGACAGGTGGCTCAAGGAAAAGAGCCTTCAGAAGGAAGGTCCAGTCGGTCATGCCTTTGCTCGGTTGATCCGCTCCCGCGACATTGTGGCGACAGCCCTG GGTCAACTGGGCCGAGATCCGTTGATCTTCCTGCATCCGCCAGAGGCGGGATGCGAAGAATGCGATGCCGCTCGCCAGTCGATTGGCTGAGCTCATGAGCGGA GAACGAGATGACGTTGGAGGGGCAAGGTCGCGCTGATTGCTGGGGCAACACGTGGAGCGGATCGGGGATTGTCTTTCTTCAGCTCCGCTTGAGACCACCCCA

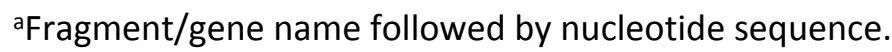

${ }^{b}$ Gene encoding tetR(B) was codon-optimized for Rhodococcus. Fragment contains Gibson Assembly overlaps.

¿Used to clone promoters, contains Gibson Assembly overlaps.

dUsed to construct uLoop recipient plasmids, contains Gibson Assembly overlaps. 
${ }^{e}$ Domesticated uLoop part, contains restriction sites and overlaps for Golden Gate Assembly.

fPromoter architecture is marked as follows: Black underlined, Gibson Assembly overlaps; Green underlined, putative -10 and -35 boxes (for $\mathrm{P}_{\mathrm{M} 6}$-derived promoters that contain overlapping promoters, TSS2 is further marker with italics); Red bold, transcriptional start site; Purple, putative extended -10 box (-TGN-); and Blue italics, tet operator sequences (tet $\mathrm{O}_{1}$ or tet $_{2}$ ). For optimized and tetO-containing promoters, changes from the parental promoter are further highlighted with Bold.

${ }^{g}$ For detailing promoter features. Marked promoters were generated by PCR as described. 
Table S5. Constitutive promoters previously used in Rhodococcus.

\begin{tabular}{|c|c|c|c|}
\hline Name ${ }^{a}$ & Use in Rhodococcus ${ }^{b}$ & Creation $^{c}$ & Characterization condition ${ }^{d}$ \\
\hline $\mathrm{P}_{\mathrm{M} 6}$ & 3 & --- & Early-stationaryb \\
\hline $\mathrm{P}_{\mathrm{T} 1}$ & 3 & --- & Early-stationary \\
\hline $\mathrm{P}_{\mathrm{T} 2}$ & 3 & --- & Early-stationary \\
\hline$P_{\text {nit }}$ & 5 & --- & Exponential $^{\mathrm{b}}$ \\
\hline$P_{\text {tet }}$ & 6 & 7 & Exponential $^{b}, n / a^{c}$ \\
\hline$P_{\text {const }}$ & 8 & 9 & Unknown $^{\mathrm{b}}$, Stationary ${ }^{\mathrm{c}}$ \\
\hline$P_{n h M}$ & 10 & --- & Unknown ${ }^{b}$ \\
\hline$P_{\text {amiM }}$ & 10 & --- & Unknown ${ }^{b}$ \\
\hline$P_{\text {smyc }}$ & 11 & 12 & Not characterized $^{b}$, Exponential $^{\mathrm{c}}$ \\
\hline$P_{\text {tac }}$ & 13 & 14 & Not characterized ${ }^{b}, n / a^{c}$ \\
\hline
\end{tabular}

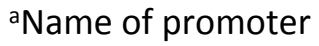

${ }^{\mathrm{b}}$ Reference for use in Rhodococcus.

'Reference for creation if different from use in Rhodococcus.

${ }^{\mathrm{d} G r o w t h}$ phase used to characterized promoters in either Rhodococcus or original host.

Table S6. Induction of tetracycline responsive promoters.

Fold-induction $^{b}$

\begin{tabular}{lcc}
\hline Promoter $^{\mathrm{a}}$ & Exponential phase & Stationary phase \\
\hline $\mathrm{P}_{\mathrm{M6-T01}}$ & $26 \pm 4$ & $30 \pm 3$ \\
$\mathrm{P}_{\mathrm{M6}-\mathrm{TO4}}$ & $3.1 \pm 0.1$ & $3.2 \pm 0.1$ \\
$\mathrm{P}_{\mathrm{M6-TO5}}$ & $46 \pm 2$ & $29 \pm 3$ \\
$\mathrm{P}_{\mathrm{M6-T07}}$ & $30 \pm 11$ & $33 \pm 5$ \\
$\mathrm{P}_{\mathrm{M6-TO9}}$ & $4.7 \pm 0.1$ & $4.0 \pm 0.2$ \\
$\mathrm{P}_{\mathrm{M6-T011}}$ & $5.0 \pm 0.6$ & $4.7 \pm 0.3$ \\
$\mathrm{P}_{\text {tet }}$ & $38 \pm 2$ & $52 \pm 3$ \\
\hline
\end{tabular}

${ }^{\text {aName of promoter }}$

${ }^{\mathrm{b}}$ Fold-induction was calculated as:

[(Induced fluorescence/OD600) - (Empty vector fluorescence/OD600]

[(Uninduced fluorescence/OD600) - (Empty vector fluorescence/OD600] 
Table S7. uLoop parts and assemblies.

\begin{tabular}{|c|c|}
\hline Plasmid & Description / Use \\
\hline \multicolumn{2}{|l|}{ Zero-level parts } \\
\hline pLZ-Pro-T1 & Zero-level plasmid containing the $\mathrm{P}_{\mathrm{T} 1}$ promoter. \\
\hline pLZ-Pro-P $\mathrm{T}_{\mathrm{T}}$ & Zero-level plasmid containing the $\mathrm{P}_{\mathrm{T} 2}$ promoter. \\
\hline pLZ-RBS-LG10 & Zero-level plasmid containing the LG-10 ribosome binding site. \\
\hline pLZ-CDS-mCherry & Zero-level plasmid containing the mCherry coding region. \\
\hline pLZ-CDS-sfGFP & Zero-level plasmid containing the sfGFP coding region. \\
\hline pLZ-Term-thcA & Zero-level plasmid containing the $\mathrm{T}_{\text {ThcA }}$ terminator. \\
\hline \multicolumn{2}{|c|}{ Odd-level assemblies } \\
\hline pLO1- $\mathrm{P}_{\mathrm{T} 1}-m$ Cherry & $\begin{array}{l}\text { Odd-level plasmid } 1 \text { containing the } \mathrm{P}_{\mathrm{T} 1} \text { promoter driving the } \\
\text { expression of } m \text { Cherry. Contains } \mathrm{LG}-10 \text { ribosome binding site } \\
\text { and the } \mathrm{T}_{\mathrm{ThCA}} \text { terminator. }\end{array}$ \\
\hline $\mathrm{pLO} 2-\mathrm{P}_{\mathrm{T} 2}-\mathrm{SfGFP}$ & $\begin{array}{l}\text { Odd-level plasmid } 2 \text { containing the } \mathrm{P}_{\mathrm{T} 2} \text { promoter driving the } \\
\text { expression of sfGFP. Contains LG-10 ribosome binding site and } \\
\text { the } \mathrm{T}_{\mathrm{ThcA}} \text { terminator. }\end{array}$ \\
\hline pLO3- $\phi C 31$ & $\begin{array}{l}\text { Odd-level plasmid } 3 \text { containing } \phi C 31 \text { integrase and it cognate } \\
\text { attP site. }\end{array}$ \\
\hline pLO4-Apra ${ }^{R}$ & $\begin{array}{l}\text { Odd-level plasmid } 4 \text { containing aac(3)-IV resistance marker for } \\
\text { apramycin. }\end{array}$ \\
\hline \multicolumn{2}{|c|}{ Even-level assemblies } \\
\hline pLEJR1 & $\begin{array}{l}\text { Even-level uLoop recipient plasmids, } 1 \text {. Constitutively co- } \\
\text { expresses } m \text { Cherry and sfGFP. Contains } \phi C 31 \text { integrase and an } \\
\text { apramycin resistance marker to allow for integration and } \\
\text { selection in Rhodococcus. }\end{array}$ \\
\hline
\end{tabular}


Zero-level parts

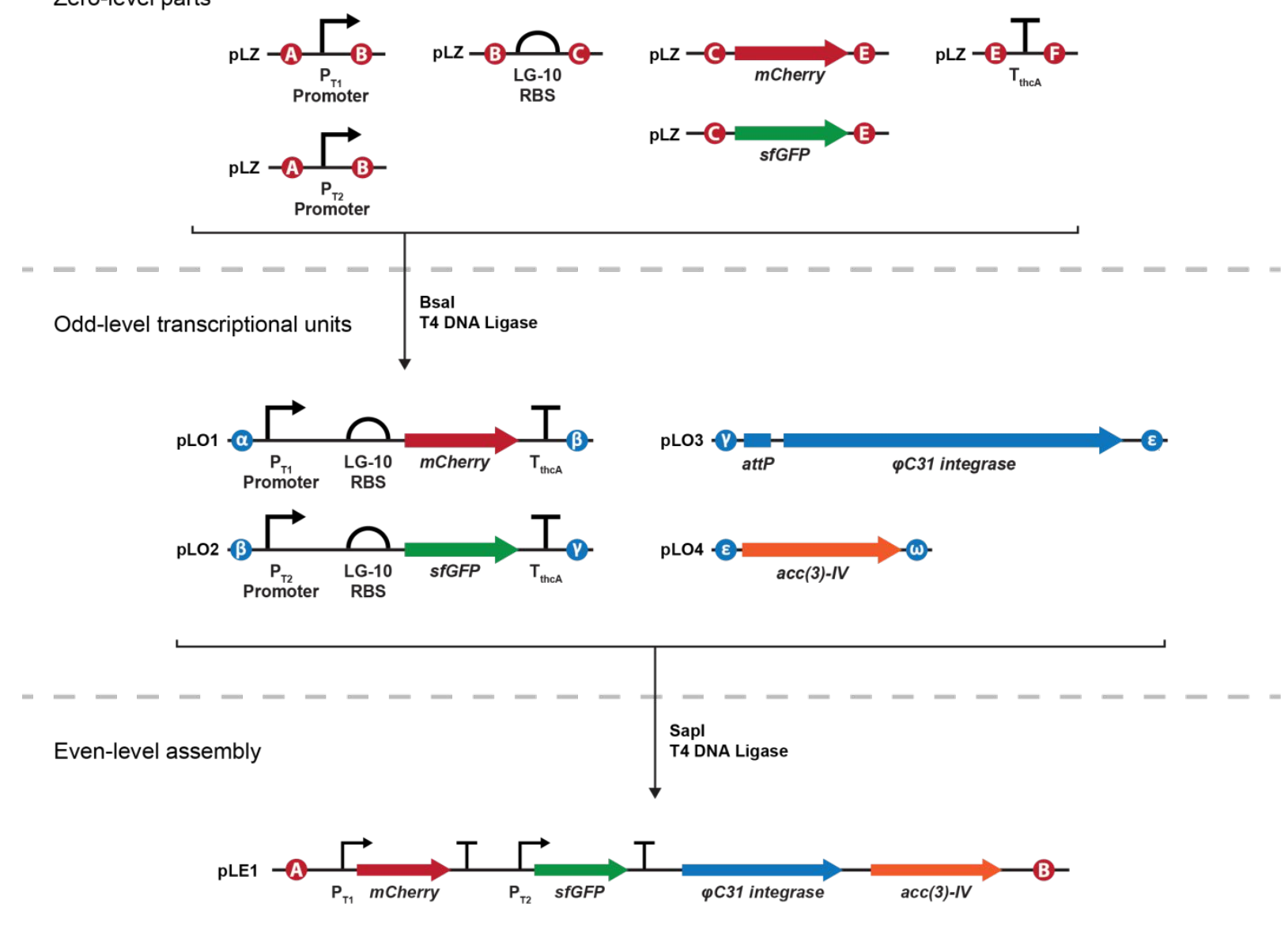

Assembly Overlaps

\begin{tabular}{|c|c|c|c|c|c|c|c|c|c|}
\hline \multicolumn{5}{|c|}{ Zero- and Even-level overlaps } & \multicolumn{5}{|c|}{ Odd-level overlaps } \\
\hline A & B & C & $\boldsymbol{E}$ & $\boldsymbol{F}$ & $\boldsymbol{\alpha}$ & B & $\boldsymbol{D}$ & ( & (1) \\
\hline GGAG & TACT & AATG & GCTT & CGCT & ATG & GCA & TAC & CAG & GGT \\
\hline
\end{tabular}

Figure S1. Schematic of uLoop-derived Golden Gate assembly strategy. 


\section{References for Supporting Information}

1. Platt, R., Drescher, C., Park, S. K., and Phillips, G. J. (2000) Genetic system for reversible integration of DNA constructs and lacZ gene fusions into the Escherichia coli chromosome, Plasmid 43, 12-23.

2. McLeod, M. P., Warren, R. L., Hsiao, W. W. L., Araki, N., Myhre, M., Fernandes, C., Miyazawa, D., Wong, W., Lillquist, A. L., Wang, D., Dosanjh, M., Hara, H., Petrescu, A., Morin, R. D., Yang, G., Stott, J. M., Schein, J. E., Shin, H., Smailus, D., Siddiqui, A. S., Marra, M. A., Jones, S. J. M., Holt, R., Brinkman, F. S. L., Miyauchi, K., Fukuda, M., Davies, J. E., Mohn, W. W., and Eltis, L. D. (2006) The complete genome of Rhodococcus sp. RHA1 provides insights into a catabolic powerhouse, Proc. Natl. Acad. Sci. USA 103, 15582-15587.

3. Round, J. W., Roccor, R., and Eltis, L. D. (2019) A biocatalyst for sustainable wax ester production: rewiring lipid accumulation in Rhodococcus to yield high-value oleochemicals, Green Chem. 21, 64686482.

4. Martínez-García, E., Goñi-Moreno, A., Bartley, B., McLaughlin, J., Sánchez-Sampedro, L., Pascual del Pozo, H., Prieto Hernández, C., Marletta, A. S., De Lucrezia, D., Sánchez-Fernández, G., Fraile, S., and de Lorenzo, V. (2019) SEVA 3.0: an update of the Standard European Vector Architecture for enabling portability of genetic constructs among diverse bacterial hosts, Nucleic Acids Res. 48, D1164-D1170.

5. Nakashima, N., and Tamura, T. (2004) Isolation and characterization of a rolling-circle-type plasmid from Rhodococcus erythropolis and application of the plasmid to multiple-recombinant-protein expression, Appl. Environ. Microbiol. 70, 5557-5568.

6. DeLorenzo, D. M., Henson, W. R., and Moon, T. S. (2017) Development of chemical and metabolite sensors for Rhodococcus opacus PD630, ACS Synth. Biol. 6, 1973-1978.

7. Rock, J. M., Hopkins, F. F., Chavez, A., Diallo, M., Chase, M. R., Gerrick, E. R., Pritchard, J. R., Church, G. M., Rubin, E. J., Sassetti, C. M., Schnappinger, D., and Fortune, S. M. (2017) Programmable transcriptional repression in mycobacteria using an orthogonal CRISPR interference platform, Nat. Microbiol. 2, 16274.

8. DeLorenzo, D. M., Rottinghaus, A. G., Henson, W. R., and Moon, T. S. (2018) Molecular toolkit for gene expression control and genome modification in Rhodococcus opacus PD630, ACS Synth. Biol. 7, 727-738.

9. Siegl, T., Tokovenko, B., Myronovskyi, M., and Luzhetskyy, A. (2013) Design, construction and characterisation of a synthetic promoter library for fine-tuned gene expression in actinomycetes, Metab. Eng. 19, 98-106.

10. Jiao, S., Yu, H., and Shen, Z. (2018) Core element characterization of Rhodococcus promoters and development of a promoter-RBS mini-pool with different activity levels for efficient gene expression, N. Biotechnol. 44, 41-49.

11. MacEachran, D. P., and Sinskey, A. J. (2013) The Rhodococcus opacus TadD protein mediates triacylglycerol metabolism by regulating intracellular NAD(P)H pools, Microb. Cell Fact. 12, 104.

12. Ehrt, S., Guo, X. V., Hickey, C. M., Ryou, M., Monteleone, M., Riley, L. W., and Schnappinger, D. (2005) Controlling gene expression in mycobacteria with anhydrotetracycline and Tet repressor, Nucleic Acids Res. 33, e21.

13. Xiong, X., Wang, X., and Chen, S. (2012) Engineering of a xylose metabolic pathway in Rhodococcus strains, Appl. Environ. Microbiol. 78, 5483-5491.

14. de Boer, H. A., Comstock, L. J., and Vasser, M. (1983) The tac promoter: a functional hybrid derived from the trp and lac promoters, Proc. Natl. Acad. Sci. USA 80, 21-25. 